\title{
BOUNDED AND COMPACT VECTORIAL HANKEL OPERATORS $\left({ }^{1}\right)$
}

\author{
BY \\ LAVON B. PAGE
}

\begin{abstract}
Operators $H$ satisfying $S^{*} H=H S$ where $S$ is a unilateral shift on Hilbert space are called Hankel operators. For a fixed shift $S$ of arbitrary multiplicity the Banach spaces of bounded Hankel operators and of compact Hankel operators are described, and it is shown that the former is always the second dual of the latter. Representations for bounded and for compact Hankel operators are given in a standard function space model.
\end{abstract}

1. Introduction. Let $\mathscr{H}$ be a separable complex Hilbert space and $S$ a shift on $\mathscr{H}$. Rosenblum [8, p. 138] has defined a Hankel operator (relative to the shift $S$ ) to be an operator $H$ on $\mathscr{H}$ satisfying the operator equation $S^{*} H=H S$. In this paper we describe completely the classes of bounded and compact Hankel operators on $\mathscr{H}$ and offer representations for such operators in a standard function-space model.

Whenever $S$ is a shift of multiplicity one, each Hankel operator $H$ may be identified with a Hankel matrix $\left[a_{m+n}\right]_{n, m=0}^{\infty}$ of complex numbers. In 1957 Nehari [7, Theorem 1] proved that if $H$ is bounded then there exists a function $f$ in $L^{\infty}$, the space of essentially-bounded complex-valued functions on the unit circle, having the property that

(i) for each nonnegative integer $n$, the $n$th Fourier coefficient of $f$ is $a_{n}$, and

(ii) $\|f\|_{\infty}=\|H\|$.

Hartman [2, p. 863] showed that the Hankel operator in question is compact if and only if there is a continuous function on the unit circle satisfying condition (i). Hevener [4] noted that Nehari's and Hartman's theorems may be used to show that the Banach space of all bounded Hankel operators relative to a simple shift is isometrically isomorphic to the second dual of the Banach space of all compact Hankel operators.

Here we shall use a recent and very elegant result of Sz-Nagy and Foiaş on extending intertwining maps between contractions [13, Theorem 2] to describe the class of bounded Hankel operators relative to a nonsimple shift. The result

Received by the editors February 6, 1969 and, in revised form, December 2, 1969.

AMS Subject Classifications. Primary 4755; Secondary 4745.

Key Words and Phrases. Bounded Hankel operator, compact Hankel operator, Toeplitz operator, shift, unitary dilation, trace-class, dual space, quotient space.

( $\left.{ }^{1}\right)$ The results of this paper were obtained in a Ph.D. dissertation written under the direction of Professor Marvin Rosenblum at the University of Virginia.

Copyright (C) 1970, American Mathematical Society 
(Theorem 2) was obtained originally by us using a dual space argument. Hartman's theorem is then generalized to allow description of compact Hankel operators, and finally it is established that the duality relationship between the classes of bounded and compact Hankel operators holds even for shifts of possibly infinite multiplicity. The study of compact Hankel operators appearing here is related to Muhly's independent research on compact operators in the commutant of a contraction [6].

Let $C$ be a separable complex Hilbert space and $L_{C}^{2}$ the space of weakly measurable functions on the unit circle to $C$ having square-integrable norm. The space $L_{C}^{2}$ and its Hardy subspace $H_{C}^{2}$ are frequently used by Helson [3, Chapter 6] and others. The standard shift on $H_{C}^{2}$ is the operator $S$ defined by $S: f\left(e^{i t}\right) \rightarrow e^{i t} f\left(e^{i t}\right)$ for $f \in H_{C}^{2}$. Representations are given for arbitrary bounded and compact Hankel operators on $H_{C}^{2}$.

2. Bounded Hankel operators. A unilateral shift $S$ is an isometry having the property that $S^{* n}$ tends strongly to zero as $n \rightarrow \infty$. Following Rosenblum [9] we fix a unilateral shift $S$ acting on a separable complex Hilbert space $\mathscr{H}$, so that

$$
\mathscr{H}=\sum_{n=0}^{\infty} \oplus S^{n} C \quad \text { where } C=(S \mathscr{H})^{\perp}
$$

[1, problem 118].

Occasionally we shall find it convenient to consider the minimal unitary extension $U$ of $S$. Thus $U$ is a bilateral shift acting on a Hilbert space $\mathscr{K}$ containing $\mathscr{H}$ which splits into the orthogonal direct sum $\mathscr{K}=\sum_{n=-\infty}^{\infty} \oplus U^{n} C$. Let $P_{+}$denote the orthogonal projection of $\mathscr{K}$ onto $\mathscr{H}$.

That such a space $\mathscr{K}$ exists is clear, as it can be constructed as a direct sum of a countable number of copies of $C$ indexed on the integers. Alternately, $U$ and $\mathscr{K}$ are obtained as the minimal unitary dilation of $S$ in the structure theory of Sz-Nagy and Foiaş [12, Chapter 1].

A frequently encountered function-space model used in studying problems related to shift operators is obtained as follows: Let $C$ denote the Hilbert space of the preceding paragraphs. Let $m$ denote normalized Lebesgue measure on [ $-\Pi, \Pi]$. Then $L_{C}^{2}$ is the Hilbert space of weakly measurable functions (technically equivalence classes of functions) defined on the unit circle, taking values in the Hilbert space $C$, and having square integrable norms. The inner product in $L_{C}^{2}$ is given by

$$
\langle f, g\rangle=\int_{-\pi}^{\pi}\left\langle f\left(e^{i t}\right), g\left(e^{i t}\right)\right\rangle d m(t) .
$$

The Hardy subspace $H_{C}^{2}$ then consists of those functions in $L_{C}^{2}$ whose negative Fourier coefficients vanish. These spaces are defined and discussed by Helson [3, Chapter 6].

The spaces $L_{C}^{2}$ and $H_{C}^{2}$ are intimately related to the spaces $\mathscr{K}$ and $\mathscr{H}$ above. 
Specifically, the mapping $\sum_{n=-\infty}^{\infty} U^{n} c_{n} \rightarrow \sum_{n=-\infty}^{\infty} c_{n} e^{i n t}$ is a unitary mapping of $\mathscr{K}$ onto $L_{C}^{2}$ which sends $\mathscr{H}$ onto $H_{C}^{2}$. More importantly, if we let $\chi$ be the identity function on the unit circle, the above unitary mapping establishes a unitary equivalence between $U$ and the multiplication operator $f \rightarrow \chi f$ on $L_{C}^{2}$. The restriction of the unitary mapping to $\mathscr{H}$ gives a unitary equivalence between the unilateral shift $S$ on $\mathscr{H}$ and the operator $f \rightarrow \chi f$ on $H_{C}^{2}$. In $\S \S 3,5$ and 6 we make extensive use of the fact that $U$ and $S$ may be realized as the operator $f \rightarrow \chi f$ on $L_{C}^{2}$ and $H_{C}^{2}$ respectively. In this section, however, we have no need for such a representation and hence continue in a model-free setting.

A bounded operator $H$ on $\mathscr{H}$ is $S$-Hankel if $S^{*} H=H S$. A bounded operator $T$ on $\mathscr{H}$ is $S$-Toeplitz if $S^{*} T S=T$; it is analytic if $S T=T S$. Since the shift $S$ is fixed, the " $S$-" of the above definitions will be dropped.

A bounded operator $L$ on $\mathscr{K}$ is called : Laurent operator (technically $L$ is $U$-Laurent) if $U L=L U$.

There is the following well-known close connection between Toeplitz and Laurent operators. For simple shifts, i.e., if $\operatorname{dim} C=1$, the result may be found in Halmos [1, problems 193 and 194], and a similar proof works for an arbitrary shift. (See solution to problem 194 in [1].)

THEOREM 1. A bounded operator $T$ on $\mathscr{H}$ is Toeplitz if and only if there exists a bounded Laurent operator $L$ on $\mathscr{K}$ such that $T$ is the projection of $L$ onto $\mathscr{H}$, i.e. $T=P_{+} L \mid \mathscr{H}$. In this case $\|L\|=\|T\|$.

The above theorem asserts that every Toeplitz operator is obtained by projecting onto $\mathscr{H}$ an operator which commutes with $U$. The next theorem establishes a similar result for Hankel operators. Every bounded Hankel operator $H$ may be obtained by projecting onto $\mathscr{H}$ an operator $J$ on $\mathscr{K}$ satisfying $U^{*} J=J U$.

THEOREM 2. A bounded operator $H$ on $\mathscr{H}$ is Hankel if and only if $H=P_{+} J \mid \mathscr{H}$ where $J$ is a bounded operator on $\mathscr{K}$ satisfying $U^{*} J=J U$.

Whenever $H$ is a bounded Hankel operator then $J$ above may be chosen so that $\|J\|=\|H\|$.

This theorem may be obtained as a special case of a very impressive theorem of Sz-Nagy and Foiaş [13, Theorem 2], one form of which is as follows: For $i=1,2$ let $W_{i}$ be a contraction on a Hilbert space $\mathscr{H}_{i}$, and let $U_{i}$ acting on the Hilbert space $\mathscr{K}_{i}$ be the minimal unitary dilation of $W_{i}$. Let $P_{i}$ be the orthogonal projection of $\mathscr{K}_{i}$ onto $\mathscr{H}_{i}$. Then a bounded operator $X$ on $\mathscr{H}_{1}$ to $\mathscr{H}_{2}$ satisfies $W_{2} X=X W_{1}$ only if there exists an operator $Y$ on $\mathscr{K}_{1}$ to $\mathscr{K}_{2}$ such that $U_{2} Y=Y U_{1},\|Y\|=\|X\|$, and $P_{2} Y P_{1}=X P_{1}$.

To obtain the nontrivial half of Theorem 2 we need only note that $U$ is the minimal unitary dilation for $S$ and $U^{*}$ is the minimal unitary dilation for $S^{*}$.

It is easily seen that the class of bounded Hankel operators on $\mathscr{H}$ forms a Banach space with the standard operator norm. Let $\mathscr{Y}$ denote this space. Let $\mathscr{T}$ denote the Banach space of all bounded Toeplitz operators on $\mathscr{H}$, and let $\mathscr{T}_{0}$ 
denote the subspace of $\mathscr{T}$ consisting of those Toeplitz operators whose range is contained in $C^{\perp}$. We describe $\mathscr{Y}$ in terms of $\mathscr{T}$ and $\mathscr{T}_{0}$.

THEOREM 3. The Banach space $\mathscr{Y}$ of bounded Hankel operators on $\mathscr{H}$ is isometrically isomorphic to the quotient space $\mathscr{T} / \mathscr{T}_{0}$.

Proof. We define an operator $R$ on $\mathscr{K}$ by $R: \sum_{n=-\infty}^{\infty} U^{n} c_{n} \rightarrow \sum_{n=-\infty}^{\infty} U^{n} c_{-n}$ whenever $c_{n} \in C$ for each $n$ and $\sum_{n=-\infty}^{\infty}\left\|c_{n}\right\|^{2}<\infty$. Then $R$ is clearly both unitary and selfadjoint.

The relation between Laurent operators and solutions $J$ to the equation

$$
U^{*} J=J U
$$

is as follows: $J$ is a solution to $\left(^{*}\right)$ if and only if $R J$ is a Laurent operator. If $J$ is a solution to $\left(^{*}\right)$, let $T_{J}$ denote the Toeplitz operator $T_{J}=P_{+} R J \mid \mathscr{H}$. For $T$ a Toeplitz operator we let $[T]$ denote the equivalence class in $\mathscr{T} / \mathscr{T}_{0}$ to which $T$ belongs.

The isometry then between $\mathscr{Y}$ and $\mathscr{T} / \mathscr{T}_{0}$ is the map which associates with each bounded Hankel operator $H$ the equivalence class $\left[T_{J}\right]$ where $H$ and $J$ are related as in Theorem 2. We note that this map is well defined; for if both $J$ and $J^{\prime}$ are related to $H$ as in Theorem 2 , then for $f \in \mathscr{H}$ and $c \in C$ we have

$$
\left\langle T_{J} f, c\right\rangle=\langle R J f, c\rangle=\langle J f, c\rangle=\langle H f, c\rangle \text { and similarly }\left\langle T_{J} f, c\right\rangle=\langle H f, c\rangle .
$$

Thus $\left\langle\left(T_{J}-T_{J^{\prime}}\right) f, c\right\rangle=0$, or $\left[T_{J}\right]=\left[T_{J^{\prime}}\right]$.

That the map is isometric and that its range is all of $\mathscr{T} / \mathscr{T}_{0}$ can be deduced from Theorem 1 and Theorem 2 . The simple observation that the map is linear completes the proof.

Note that to this point separability of the underlying Hilbert space $\mathscr{H}$ has not been invoked. The separability of $\mathscr{H}$ permits $\mathscr{T}$ and $\mathscr{T}_{0}$ to be described as spaces of weakly measurable operator-valued functions. Let $L^{\infty}[B(C)]$ denote the algebra of essentially-bounded functions from the unit circle to the set $B(C)$ of bounded operators on the Hilbert space $C$. Let $H_{0}^{\infty}[B(C)]$ denote the subspace of $L^{\infty}[B(C)]$ consisting of those operator-valued functions whose nonpositive Fourier coefficients vanish.

COROLlaRY. The space $\mathscr{Y}$ is isometrically isomorphic to the quotient space $L^{\infty}[B(C)] / H_{0}^{\infty}[B(C)]$.

Proof. The space $\mathscr{T}$ and $L^{\infty}[B(C)]$ are isometrically isomorphic under an isomorphism that carries $\mathscr{T}_{0}$ onto $H_{0}^{\infty}[B(C)]$. This follows from Theorem 1 and a standard argument which shows that every bounded Laurent operator on $\mathscr{K}$ is unitarily equivalent to multiplication by an $L^{\infty}[B(C)]$ function in the model $L_{C}^{2}$.

We will indicate that the preceding results yield a solution to a related extremal problem. It will first be necessary to discuss briefly linear transformations which are "analytic" but unbounded. 
Any operator $A$ on $C$ can be extended "analytically" so that its domain includes all "analytic trigonometric polynomials," i.e. all $f \in \mathscr{H}$ of the form $f=\sum_{n=0}^{N} S^{n} c_{n}$ where $N$ is a positive integer and $c_{n} \in C$ for $n=0,1, \ldots, N$, by defining

$$
A: \sum_{n=0}^{N} S^{n} c_{n} \rightarrow \sum_{n=0}^{N} S^{n} A c_{n} .
$$

The transformation $A$ then is densely defined in $\mathscr{H}$ but in general unbounded. The following properties are obviously satisfied:

(i) The domain of $A$ contains $C$ and is invariant under $S$.

(ii) If $f$ is in the domain of $A$, then $S A f=A S f$.

(iii) The restriction of $A$ to $C$ is a bounded operator.

An operator on $\mathscr{H}$ which satisfies these conditions will be called an analytic transformation.

LEMMA 1. If $A$ is any analytic transformation, then the domain of $A^{*}$ includes all analytic trigonometric polynomials, and $A^{*}$ is bounded on $C$.

Proof. For each nonnegative integer $N$, let $P_{N}$ be the orthogonal projection of $\mathscr{H}$ onto $\sum_{n=0}^{N} \oplus S^{n} C$, and let $A_{N}=P_{N} A$. Then $A_{N}$ is bounded. Suppose that $g=\sum_{n=0}^{M} S^{n} c_{n}$ is an analytic trigonometric polynomial. Then if $f$ is any element in the domain of $A$,

$$
\langle A f, g\rangle=\left\langle A f, P_{M} g\right\rangle=\left\langle P_{M} A f, g\right\rangle=\left\langle A_{M} f, g\right\rangle=\left\langle f, A_{M}^{*} g\right\rangle .
$$

Thus $g$ is in the domain of $A^{*}$ and $A^{*} g=A_{M}^{*} g$. In particular $A^{*} c=A_{1}^{*} c$ for $c \in C$, and hence $A^{*}$ is bounded on $C$.

Of interest to us are analytic transformations obtained by first restricting a bounded Hankel operator to $C$ and then extending this restriction analytically. The following theorem indicates that the norm of the Hankel operator occurs as the solution to an extremal problem involving the analytic transformation.

THEOREM 4. Let $H$ be a bounded Hankel operator on $\mathscr{H}$ and let $A$ be an analytic transformation which agrees with $H$ on the subspace $C$.

Then $\|H\|=\inf _{B}\left\|A+B^{*}\right\|$ where the infimum is taken over the set of all analytic transformations with range contained in $C^{\perp}$. There exists a transformation $B$ for which the infimum is achieved.

(Note. For any $B$ the domain of $A+B^{*}$ is dense by the lemma.)

Proof. Suppose $B$ is an analytic transformation with range contained in $C^{\perp}$ and such that $A+B^{*}$ is bounded.

Since $A+B^{*}$ is Toeplitz, by Theorem 1 it follows that $A+B^{*}$ is the projection onto $\mathscr{H}$ of a Laurent operator $L$ defined on $\mathscr{K}$, and $\|L\|=\left\|A+B^{*}\right\|$. It is easily checked that on the dense set of analytic trigonometric polynomials $H$ agrees with $P_{+} R L^{*}$ where $R$ is as in the proof of Theorem 3. Thus $\|H\| \leqq\left\|A+B^{*}\right\|$. 
The existence of an analytic transformation $B$ for which equality holds in the inequality $\|H\| \leqq\left\|A+B^{*}\right\|$ follows from Theorem 2 . For by Theorem 2 there exists an operator $J$ on $\mathscr{K}$ with $U^{*} J=J U$ and $\|J\|=\|H\|$.

Define $B$ on the dense set of analytic trigonometric polynomials by $B=P_{+} R J^{*}$ $-A^{*}$. It is a routine matter to verify that $B$ so defined commutes with the shift $S$ and has range contained in $C^{\perp}$. Finally, $\|H\|=\|J\|=\left\|J^{*}\right\| \geqq\left\|A+B^{*}\right\|$, so in fact $\|H\|=\left\|A+B^{*}\right\|$.

Theorem 4 can be restated as a solution to an extremal problem concerning operator-valued analytic functions defined in the unit disk. Such a statement is the operator-valued analogue of the Nehari theorem [7, Theorem 1].

3. A representation theorem for bounded Hankel operators. Recall that $\chi$ is the identity function on the unit circle, $\chi\left(e^{i t}\right)=e^{i t}$. We now make use of the fact that the bilateral shift $U$ and the unilateral shift $S$ of the preceding section may be realized as multiplication by $\chi$ on $L_{C}^{2}$ and $H_{C}^{2}$ respectively, and in this section we use the symbols $U$ and $S$ to denote the aforementioned multiplication operators. Thus a bounded operator $H$ on $H_{C}^{2}$ will be Hankel if and only if $S^{*} H=H S$ where $S f=\chi f$.

We now give a description of the completely general bounded Hankel operator on $H_{C}^{2}$.

THEOREM 5. A bounded operator $H$ on $H_{C}^{2}$ is Hankel if and only if there exists a function $F$ in $L^{\infty}[B(C)]$ such that

$$
\langle H f, g\rangle=\int_{-\pi}^{\pi}\left\langle F\left(e^{i t}\right) f\left(e^{-i t}\right), g\left(e^{i t}\right)\right\rangle d m(t) \quad \text { for all } f, g \in H_{C}^{2} .
$$

If $H$ is a bounded Hankel operator on $H_{C}^{2}$ then the function $F$ in $L^{\infty}[B(C)]$ may be chosen so that $\|F\|_{\infty}=\|H\|$.

Proof. In this setting the extension space $\mathscr{K}$ is just $L_{C}^{2}$ and $U$ is the operator defined by $U: f \rightarrow \chi f$ for all $f$ in $L_{C}^{2}$. By Theorem 2 there exists an operator $J$ on $L_{C}^{2}$ such that the projection of $J$ onto $H_{C}^{2}$ is $H, U^{*} J=J U$, and $\|J\|=\|H\|$. Using the known fact that the commutant of $U$ consists of all multiplications by $L^{\infty}[B(C)]$ functions, it is not difficult to check that any such operator $J$ must be of the form

$$
J: f\left(e^{i t}\right) \rightarrow F\left(e^{i t}\right) f\left(e^{-i t}\right) \quad \text { for } f \in L_{C}^{2},
$$

and that $\|J\|=\|F\|_{\infty}$. Thus $\|H\|=\|F\|_{\infty}$ and

$$
\langle H f, g\rangle=\int_{-\pi}^{\pi}\left\langle F\left(e^{i t}\right) f\left(e^{-i t}\right), g\left(e^{i t}\right)\right\rangle d m(t) .
$$

4. Compact Hankel operators. In $\S 2$ the Banach space $\mathscr{Y}$ of bounded Hankel operators was studied. Now we describe the Banach space of compact Hankel operators on $\mathscr{H}$ which we denote by $\mathscr{Y}_{0}$. 
As a preliminary we fix the additional notation used in this section. We will denote by $\mathfrak{S}_{1}$ the ideal of trace-class operators in the algebra $B(C)$ of bounded operators on $C$. If $B \in \mathfrak{S}_{1}$, we denote the $\mathfrak{S}_{1}$-norm of $B$ by $\|B\|_{1}$. The ideal of compact operators in $B(C)$ we denote by $\mathfrak{S}_{\infty}$.

A Banach space which is of fundamental importance in the discussion of compact Hankel operators is the space of all functions defined on the unit circle and taking values in $\mathfrak{S}_{\infty}$ which are continuous relative to the uniform operator topology on $\Im_{\infty}$. This linear space is a Banach space under the sup norm and will be denoted here by $C\left(\Im_{\infty}\right)$. We let $C^{+}\left(\Im_{\infty}\right)=C\left(\Im_{\infty}\right) \cap H_{0}^{\infty}[B(C)]$, the subspace of $C\left(\Im_{\infty}\right)$ consisting of those functions whose nonpositive Fourier coefficients vanish.

The Banach space $\mathscr{Y}_{0}$ of compact Hankel operators is described in terms of $C\left(\mathfrak{S}_{\infty}\right)$ just as $\mathscr{Y}$, the space of bounded Hankel operators, is described in terms of $L^{\infty}[B(C)]$ in the corollary to Theorem 3 .

THEOREM 6. The Banach space $\mathscr{Y}_{0}$ of compact Hankel operators on $\mathscr{H}$ is isometrically isomorphic to the quotient space $C\left(\mathfrak{S}_{\infty}\right) / C^{+}\left(\mathfrak{S}_{\infty}\right)$.

Theorem 6 will be proved in the next section. With the description of $\mathscr{Y}_{0}$ provided by Theorem 6 we can now examine the duality relationships existing between the spaces considered above.

Let $L^{1}\left(\Im_{1}\right)$ denote the space of all weakly measurable operator-valued functions $F$ on the unit circle with values in $\Im_{1}$ satisfying

$$
\|F\|_{L^{1}\left(\mathfrak{S}_{1}\right)}=\int_{-\pi}^{\pi}\left\|F\left(e^{i t}\right)\right\|_{1} d m(t)<\infty .
$$

This space is defined by Sarason [11] who states the following proposition and notes that the proof is an easy corollary to the analogous well-known theorem for scalar-valued functions.

TheOREM 7. The space $L^{\infty}[B(C)]$ is the dual of $L^{1}\left(\Im_{1}\right)$. An element $Q$ in $L^{\infty}[B(C)]$ corresponds to the linear functional

$$
F \rightarrow \int_{-\pi}^{\pi} \operatorname{tr}\left[Q\left(e^{i t}\right) F\left(e^{i t}\right)\right] d m(t), \quad F \in L^{1}\left(\Im_{1}\right) .
$$

We denote by $H^{1}\left(\Im_{1}\right)$ the subspace of $L^{1}\left(\Im_{1}\right)$ consisting of those functions in $L^{1}\left(\Im_{1}\right)$ whose negative Fourier coefficients vanish. It is not difficult to verify that $H_{0}^{\infty}[B(C)]$ is the annihilator in $L^{\infty}[B(C)]$ of the subspace $H^{1}\left(\Im_{1}\right)$ of $L^{1}\left(\Im_{1}\right)$. Then it follows that $L^{\infty}[B(C)] / H_{0}^{\infty}[B(C)]$ is the dual space of $H^{1}\left(\Im_{1}\right)$.

The next theorem completes the picture by describing $H^{1}\left(\Im_{1}\right)$ as the dual space of $C\left(\Im_{\infty}\right) / C^{+}\left(\Im_{\infty}\right)$ and thus yielding Theorem 9 as an immediate consequence.

THEOREM 8. The space $H^{1}\left(\Im_{1}\right)$ is the dual space of $C\left(\Im_{\infty}\right) / C^{+}\left(\Im_{\infty}\right)$.

The correspondence is the following: Let $[F]$ denote the equivalence class in the 
above quotient space to which a function $F$ in $C\left(\mathfrak{S}_{\infty}\right)$ belongs. A function $G$ in $H^{1}\left(\mathfrak{S}_{1}\right)$ then corresponds to the linear functional

$$
F \rightarrow \int_{-\pi}^{\pi} \operatorname{tr}\left[G\left(e^{i t}\right) F\left(e^{i t}\right)\right] d m(t), \quad F \in C\left(\widetilde{S}_{\infty}\right) .
$$

Ryan [10] offers an account of the study of dual spaces of spaces of continuous functions from a compact Hausdorff space to a Banach space $X$. These results describe the dual as a space of vector-valued measures taking values in $X^{*}$.

We are interested in the space $C\left(\Im_{\infty}\right)$. The conclusion of Ryan's Theorem 1 in the present context is as follows: The annihilator in $\left[C\left(\mathfrak{S}_{\infty}\right)\right]^{*}$ of $C^{+}\left(\mathfrak{S}_{\infty}\right)$ is the space of all bounded linear functionals of the form

$$
F \rightarrow \int_{-\pi}^{\pi} \operatorname{tr}\left[F\left(e^{i t}\right) G\left(e^{i t}\right)\right] d m(t), \quad F \in C\left(\Im_{\infty}\right) \text { where } G \text { is in } H^{1}\left(\Im_{1}\right) .
$$

The norm of such a linear functional is equal to $\int_{-\pi}^{\pi}\left\|G\left(e^{i t}\right)\right\|_{1} d m(t)$. But for any Banach space $X$ with closed subspace $X_{0},\left(X / X_{0}\right)^{*}$ is isometrically isomorphic to the annihilator of $X_{0}$ in $X^{*}$. Letting $X=C\left(\Im_{\infty}\right)$ and $X_{0}=C^{+}\left(\Im_{\infty}\right)$ we obtain Theorem 8.

THEOREM 9. The space $\mathscr{Y}$ is isometrically isomorphic to the second dual space of $\mathscr{Y}$.

5. Proof of Theorem 6. Theorem 6 will follow from the four lemmas below which also yield the representation theorem for compact Hankel operators appearing in $\S 6$. In this section we take the model $H_{C}^{2}$ of $\S 3$ as the underlying space so that $\mathscr{Y}$ and $\mathscr{Y}_{0}$ are now considered to be the classes of bounded and compact Hankel operators on $H_{C}^{2}$.

Lemma 2. Suppose $F \in C\left(\mathfrak{S}_{\infty}\right)$ and $H$ is an operator on $H_{C}^{2}$ satisfying

$$
\langle H f, g\rangle=\int_{-\pi}^{\pi}\left\langle F\left(e^{i t}\right) f\left(e^{-i t}\right), g\left(e^{i t}\right)\right\rangle d m(t), \quad f, g \in H_{C}^{2} .
$$

Then $H$ is a compact Hankel operator.

Proof. Set $W_{j}=\int_{-\pi}^{\pi} F\left(e^{i t}\right) e^{-i j t} d m(t)$. Thus $W_{j}$, being a norm limit of compact Riemann sums, is compact.

We may extend $F$ continuously to the closed unit disk so that $F\left(r e^{i t}\right)=$ $\sum_{j=-\infty}^{\infty} r^{|j|} e^{i j t} W_{j}$ where the series converges (in operator norm) uniformly on compact subsets of the open unit disk.

Define $F_{p}(z)=F(p z)$ for $0<p<1$ and $z \leqq 1$, and $F_{p}^{(n)}\left(r e^{i t}\right)=\sum_{j=-n}^{n} p^{|j|} r^{|j|} e^{i j t} W_{j}$ for each positive integer $n$.

Now define Hankel operators $H_{p}$ and $H_{p}^{(n)}$ for $0<p<1$ and $n$ a positive integer by

$$
\left\langle H_{p} f, g\right\rangle=\int_{-\pi}^{\pi}\left\langle F_{p}\left(e^{i t}\right) f\left(e^{-i t}\right), g\left(e^{i t}\right)\right\rangle d m(t) \quad \text { for } f, g \in H_{C}^{2}
$$




$$
\left\langle H_{p}^{(n)} f, g\right\rangle=\int_{-\pi}^{\pi}\left\langle F_{p}^{(n)}\left(e^{i t}\right) f\left(e^{-i t}\right), g\left(e^{i t}\right)\right\rangle d m(t) \quad \text { for } f, g \in H_{C}^{2} .
$$

Notice that $\chi^{n+1} H_{C}^{2}$ is contained in the kernel of $H_{p}^{(n)}$, and if $0 \leqq j+k \leqq n$ and $c_{1}$, $c_{2} \in C$,

$$
\left\langle H_{p}^{(n)} \chi^{j} c_{1}, \chi^{k} c_{2}\right\rangle=p^{j+k}\left\langle W_{j+k} c_{1}, c_{2}\right\rangle .
$$

(The term on the left is zero if $j+k>n$ since $\left\langle H_{p}^{(n)} \chi^{j} c_{1}, \chi^{k} c_{2}\right\rangle=\left\langle H_{p}^{(n)} \chi^{j+k} c_{1}, c_{2}\right\rangle$.) Thus $H_{p}^{(n)}$ is a finite sum of compact operators and is hence compact.

For a fixed $p, 0<p<1, F_{p}^{(n)}$ converges uniformly to $F_{p}$ as $n \rightarrow \infty$, and so from (1) and (2) above we deduce that $\left\|H_{p}-H_{p}^{(n)}\right\| \rightarrow 0$ as $n \rightarrow \infty$ and thus $H_{p}$ is compact.

Finally, a standard approximate identity argument (Hoffman [5, p. 17]) shows that $F_{p} \rightarrow F$ uniformly as $p \rightarrow 1-$.

But $\left\|H_{p}-H\right\| \leqq \sup _{t}\left\|F_{p}\left(e^{i t}\right)-F\left(e^{i t}\right)\right\|$, and so $\left\|H_{p}-H\right\| \rightarrow 0$ and $H$ is compact.

LEMMA 3. The transformation $[F] \rightarrow H$ where $F$ and $H$ are related by

$$
\langle H f, g\rangle=\int_{-\pi}^{\pi}\left\langle F\left(e^{-i t}\right) f\left(e^{-i t}\right), g\left(e^{i t}\right)\right\rangle d m(t), \quad f, g \in H_{C}^{2},
$$

is an isometry of the Banach space $C\left(\mathfrak{S}_{\infty}\right) / C^{+}\left(\mathfrak{S}_{\infty}\right)$ into the Banach space $\mathscr{Y}_{0}$.

Proof. If $F \in C^{+}\left(\Im_{\infty}\right)$ then $\int_{-\pi}^{\pi}\left\langle F\left(e^{-i t}\right) f\left(e^{-i t}\right), g\left(e^{i t}\right)\right\rangle d m(t)=0$ for all $f, g \in H_{C}^{2}$. Thus the above transformation is well defined. Its range is contained in $\mathscr{Y}_{0}$ by Lemma 2.

Clearly if $H$ and $F$ are related by $\left(^{*}\right)$ then $\|H\| \leqq \sup _{t}\left\|F\left(e^{i t}\right)\right\|$. If $F_{1} \in C\left(\Im_{\infty}\right)$ and $F-F_{1} \in C^{+}\left(\Im_{\infty}\right)$ then $\left(^{*}\right)$ holds with $F$ replaced by $F_{1}$, and hence $\|H\|$ $\leqq \sup _{t}\left\|F_{1}\left(e^{i t}\right)\right\|$. Thus $\|H\| \leqq\|[F]\|$ where the term on the right is the norm in the quotient space. We must yet establish the reverse inequality.

We continue to assume that $H$ and $F$ are related by $\left({ }^{*}\right)$ where $F \in C\left(\Im_{\infty}\right)$. Theorem 5 offers an alternate representation for $H$. It asserts that there exists a function $F_{1}$ in $L^{\infty}[B(C)]$ such that

$$
\langle H f, g\rangle=\int_{-\pi}^{\pi}\left\langle F_{1}\left(e^{-i t}\right) f\left(e^{-i t}\right), g\left(e^{i t}\right)\right\rangle d m(t) \quad \text { for } f, g \in H_{C}^{2}
$$

and such that $\|H\|=$ ess-sup $_{t}\left\|F\left(e^{i t}\right)\right\|$. But then

$$
\int_{-\pi}^{\pi}\left\langle\left[F\left(e^{-i t}\right)-F_{1}\left(e^{-i t}\right)\right] f\left(e^{-i t}\right), g\left(e^{i t}\right)\right\rangle d m(t)=0 \quad \text { for all } f, g \in H_{C}^{2},
$$

from which it follows that $F-F_{1}$ is in $H_{0}^{\infty}[B(C)]$.

Recall now that any Banach space may be embedded isometrically in its second dual in a natural way. Theorem 8 , used in conjunction with the remark immediately following Theorem 7 , shows that $L^{\infty}[B(C)] / H_{0}^{\infty}[B(C)]$ is the second dual of $C\left(\Im_{\infty}\right) / C^{+}\left(\Im_{\infty}\right)$. An examination of the manner in which the dual spaces act indicates that the natural embedding of $C\left(\Im_{\infty}\right) / C^{+}\left(\Im_{\infty}\right)$ is as follows: If $G$ is a 
function in $C\left(\Im_{\infty}\right)$, then the coset $G+C^{+}\left(\Im_{\infty}\right)$ is sent into the coset $G+H_{0}^{\infty}[B(C)]$. Since the natural embedding is isometric, it follows that the cosets $G+C^{+}\left(\Im_{\infty}\right)$ and $G+H_{0}^{\infty}[B(C)]$ have the same norms in their respective spaces.

Returning now to $F$ and $F_{1}$ above, since $F$ and $F_{1}$ are in the same coset in $L^{\infty}[B(C)] / H_{0}^{\infty}[B(C)]$ and since $\left\|F_{1}\right\|_{\infty}=\|H\|$, it follows that the norm of the coset $F+H_{0}^{\infty}[B(C)]$ is not greater than $\|H\|$. But by the general remarks in the preceding paragraph, this implies that the norm of the coset $F+C^{+}\left(\Im_{\infty}\right)$ is not greater than $\|H\|$, i.e. $\|[F]\| \leqq\|H\|$.

The techniques used in Lemma 3 were used by Hevener [4]. Lemma 4 is essentially due to Hartman. Hartman's proof [2 Lemma 2] is for scalar Hankel operators but applies just as well in the present setting.

LEMMA 4. Suppose $H$ is a compact Hankel operator on $H_{C}^{2}$ and

$$
\langle H f, g\rangle=\int_{-\pi}^{\pi}\left\langle F\left(e^{i t}\right) f\left(e^{-i t}\right), g\left(e^{i t}\right)\right\rangle d m(t) \quad \text { for } f, g \in H_{C}^{2}
$$

where $F$ is in $L^{\infty}[B(C)]$.

For $0<p<1$, let $F_{p}\left(e^{i t}\right)=F\left(p e^{i t}\right)$. (It is assumed here that $F$ has been extended to the unit disk in the natural manner via the Poisson kernel as in [5].) Let $H_{p}$ be defined for $0<p<1$ by

$$
\left\langle H_{p} f, g\right\rangle=\int_{-\pi}^{\pi}\left\langle F_{p}\left(e^{i t}\right) f\left(e^{-i t}\right), g\left(e^{i t}\right)\right\rangle d m(t) \quad \text { for } f, g \in H_{C}^{2} .
$$

Then $H_{p}$ converges to $H$ in operator norm as $p \rightarrow 1-$.

LEMMA 5. The range of the transformation of Lemma 3 is $\mathscr{Y}_{0}$.

Proof. Let $H \in \mathscr{Y}_{0}$. By Theorem 5 there exists a function $F$ in $L^{\infty}[B(C)]$ such that $H$ and $F$ are related as in Lemma 4. Let $H_{p}$ be as in Lemma 4 for $0<p<1$.

For each integer $n$, let $W_{n}$ be the $n$th Fourier coefficient of $F$. Thus if $c_{1}, c_{2} \in C$,

$$
\left\langle W_{n} c_{1}, c_{2}\right\rangle=\int_{-\pi}^{\pi}\left\langle F\left(e^{i t}\right) c_{1}, c_{2}\right\rangle e^{-i n t} d m(t) .
$$

If $n \geqq 0,\left\langle W_{n} c_{1}, c_{2}\right\rangle=\left\langle H c_{1}, \chi^{n} c_{2}\right\rangle$ and hence $W_{n}$ is compact.

The function $F_{p}$ is given by $F_{p}\left(e^{i t}\right)=\sum_{n=-\infty}^{\infty} p^{|n|} e^{i n t} W_{n}$. Let $G_{p}$, for $0<p<1$, be the function defined by

$$
G_{p}\left(e^{i t}\right)=\sum_{n=0}^{\infty} p^{n} e^{i n t} W_{n} .
$$

Since $W_{n}$ is compact for $n \geqq 0$ and since the series converges in the uniform operator topology, it follows that $G_{p} \in C\left(\Im_{\infty}\right)$. Also

$$
\left\langle H_{p} f, g\right\rangle=\int_{-\pi}^{\pi}\left\langle G_{p}\left(e^{i t}\right) f\left(e^{-i t}\right), g\left(e^{i t}\right)\right\rangle d m(t) \quad \text { for } f, g \in H_{C}^{2} .
$$

Thus the transformation of Lemma 3 sends $\left[G_{p}\left(e^{-i t}\right)\right]$ onto $H_{p}$. 
We have established that each $H_{p}, 0<p<1$, is in the range of the transformation of Lemma 3. The transformation is already known to be isometric, and hence its range is closed. From Lemma 4 it now follows that $H$ is in the range.

6. A representation theorem for compact Hankel operators. The above lemmas which have been used to prove Theorem 6 also yield a representation for the compact Hankel operators on $H_{C}^{2}$.

THEOREM 10. A bounded operator $H$ on $H_{C}^{2}$ is a compact Hankel operator if and only if there exists a function $F$ in $C\left(\Im_{\infty}\right)$ such that

$$
\langle H f, g\rangle=\int_{-\pi}^{\pi}\left\langle F\left(e^{i t}\right) f\left(e^{-i t}\right), g\left(e^{i t}\right)\right\rangle d m(t) \quad \text { for } f, g \in H_{C}^{2} .
$$

If $H$ is compact Hankel and $\varepsilon>0$, then $F$ may be chosen so that

$$
\|H\| \leqq \sup _{t}\left\|F\left(e^{i t}\right)\right\| \leqq\|H\|+\varepsilon .
$$

\section{REFERENCES}

1. P. R. Halmos, A Hilbert space problem book, Van Nostrand, Princeton, N. J., 1967. MR 34 \#8178.

2. P. Hartman, On completely continuous Hankel matrices, Proc. Amer. Math. Soc. 9 (1958), 862-866. MR 21 \#7399.

3. H. Helson, Lectures on invariant subspaces, Academic Press, New York, 1964. MR 30 \#1409.

4. R. N. Hevener, A functional analytic approach to Hankel and Toeplitz matrices, Thesis, Univ. of Virginia, Charlottesville, 1965.

5. K. Hoffman, Banach spaces of analytic functions, Prentice-Hall Series in Modern Analysis, Prentice-Hall, Englewood Cliffs, N. J., 1962. MR 24 \#A2844.

6. P. S. Muhly, Commutants containing a compact operator, Bull. Amer. Math. Soc. 75 (1969), 353-356.

7. Z. Nehari, On bounded bilinear forms, Ann. of Math. (2) 65 (1957), 153-162. MR 18, 633.

8. M. Rosenblum, Self-adjoint Toeplitz operators, Summer Institute of Spectral Theory and Statistical Mechanics 1965, Brookhaven National Laboratory, Upton, New York, 1966. MR 34 \#4084.

9. - Vectorial Toeplitz operators and the Fejér-Riesz theorem, J. Math. Anal. Appl. 23 (1968), 139-147. MR 37 \#3378.

10. R. Ryan, The F. and $M$. Riesz theorem for vector measures, Nederl. Akad. Wetensch. Proc. Ser. A66=Indag. Math. 25 (1963), 408-412. MR 27 \#2848.

11. D. Sarason, Generalized interpolation in $H^{\infty}$, Trans. Amer. Math. Soc. 127 (1967), 179-203. MR 34 \#8193.

12. B. Sz-Nagy and C. Foiaş, Analyse harmonique des opérateurs de l'espace de Hilbert, Masson, Paris and Akad. Kiadó, Budapest, 1967. MR 37 \#778.

13. — Dilatation des commutants d'opérateurs, C. R. Acad. Sci. Paris Sér. A-B 266 (1968), A493-A495. MR 38 \#5049.

UNIVERSITY OF VIRGINIA,

Charlottesville, Virginia 22901

North Carolina State University, Raleigh, North Carolina 27607 